\title{
Enhancing Face Recognition Using PC-SIFTTechnique: A Hybrid Approach
}

\author{
Deepti Ahlawat ${ }^{1 *}$ and Vijay Nehra ${ }^{1}$ \\ ${ }^{1}$ Department of Electronics and Communication Engineering \\ Bhagat Phool Singh Mahila Vishwavidyalaya, Khanpur Kalan, Sonipat, \\ Haryana, India \\ *deeptijaglan@gmail.com,nehra_vijay@yahoo.co.in
}

\begin{abstract}
In this investigation, a hybrid approach using phase congruency $(P C)$ and Shift Invariant Feature Transform (SIFT) for face recognition is presented. The present study exploits the unique advantages of both phase congruency and Shift Invariant Feature Transform and makes it an effective feature extraction technique. Support Vector Machine $(S V M)$ is used for the classification. The effectiveness of the present work is verified and compared by using another classifier i.e. K-Means. The results of this study shows that $P C$ - SIFT are more robust to expression variations and shows better results than other methods. The present approach achieves good recognition accuracy on Japanese Female Facial Expression (JAFFE) and Cohn Kanade (CK) database. From the experiments it is found that PC-SIFT outperforms SIFT technique and provide an average recognition accuracy of $92.4 \%$ and $91.5 \%$ respectively for different expression for JAFFE and CK datasets. The proposed technique has been compared with the state-of-art techniques and it is observed that proposed approach gives better results than existing techniques.
\end{abstract}

Keywords: Adaboost, Phase Congruency, Shift Invariant Feature Transform, Face Recognition

\section{Introduction}

Face Recognition is one of the intensive research topic in the field of image processing, computer vision and multimedia. Recognition of face is something that can be done effortlessly, yet it has remained a difficult problem in field of image processing. Human face plays a significant role in identification and verification process and compared to machine, human can identify faces at much faster rate. Face recognition as a biometric has a number of advantages which makes it the primary biometric that human use to recognize a person. Though human beings can detect and identify faces in a cluttered scene with very little effort, but building an automated system that accomplishes such tasks is very challenging. In last two decades face recognition has found a number of applications in public identification and security, verification of driving license and credit cards, passport verification, criminal justice system, forensic and law enforcement applications [1-4]. Human brain has limitations that it cannot remember a large number of people accurately after a long time but the advantage of computer system is that, it can handle a large set of face images with ease. But one of the major drawbacks of a face recognition system is its relative low accuracy due to poorly extracted features which leads to poor recognition rate.

A good feature extraction technique will improve the performance of face recognition system. The authors in literature [5-6] say that the various techniques exist for feature

Received (February 28, 2017), Review Result (June 26, 2017), Accepted (July 8, 2017) 
extraction. Out of those methods feature extraction techniques are classified into two groups: representation of features by the local regions [7-8] and by the global method [9]. The most widely used global method for feature extraction is Principal Component Analysis (PCA) [10]. It extracts features from whole face image which are represented by eigenfaces derived from the eigen vectors from covariance matrix. The linear combination weights for eigenfaces are calculated which represents the face. PCA is simple and fast method but has problems in recognition under illumination and pose variation [7]. In 2004, D. Lowe [11] proposed Scale Invariant Feature Transform (SIFT) algorithm which was widely used in subject detection and recognition issues. The advantages of this method are 1) SIFT features are invariant to rotation, scale and illumination; 2) SIFT features maintains a degree of stability and robustness to noise [12-13]. PC is used for edge detection and the main aim of this technique is to identify and locate sharp discontinuities in an image. Specially in images, phase conveys more information than magnitude, also phase is more immune to noise and contrast distortions [17]. Keeping in view the unique features of PC and SIFT, a hybrid work is proposed. In this investigation, SIFT technique along with phase congruency (PC) technique is used to enhance the extracted features and improve the results in terms of recognition accuracy. The results are classified with the SVM classifier and K-Means classifier.

In nutshell, the present investigation is an attempt made to apply hybrid PC-SIFT based feature extraction technique for face recognition. The rest of the paper is organized as follows: Section 2 describes the previous work related to feature extraction techniques. Section 3 discusses the framework for present investigation. Section 4 presents the experimental results and discussion followed by conclusion.

\section{Related Work}

In this section, a brief overview of literature is presented in Table 1.

Table 1. Overview of Related Work

\begin{tabular}{|c|c|}
\hline Techniques & Researchers \\
\hline $\begin{array}{l}\text { Phase Congruency } \\
\text { PC) }\end{array}$ & $\begin{array}{l}\text { Morrone et al.(1988); Venkatesh et al. (1998); Kovesi et al. (1999); } \\
\text { Yuan et al. (2005); Skarbnik et al.(2009); Struc et al. (2009), Yang et } \\
\text { al. (2017) }\end{array}$ \\
\hline $\begin{array}{l}\text { Shift Invariant Feature } \\
\text { Transform (SIFT) }\end{array}$ & $\begin{array}{l}\text { Lowe et al.(2004); Ke et al. (2004); Pun et al. (2004); Lin et al.(2011); } \\
\text { Wang et al. (2013); Dagher et al.(2014); Krizaj et al. (2014); Luo et } \\
\text { al. (2015). }\end{array}$ \\
\hline
\end{tabular}

Literature survey of [5-13] shows that, there are various techniques available for feature extraction. Out of these techniques, Lowe in 2004 first proposed the technique for face recognition based on Scale Invariant Feature Transform whose main idea was to detect feature points efficiently [11].

For face detection PC is used along with SIFT, PC was used as a local energy model for edge detection by Morrone and Blurr [15] which was further used for feature extraction [16]. Kovesi in his work further modified and extended the work to 2 dimensions over different scales and orientations [17]. 
Keeping in view the literature survey and above facts, the objective of present investigation is to utilize PC-SIFT feature extraction technique for face recognition and compare the results using SVM and K-Means classifier on JAFFE and CK datasets. After a brief overview of the literature survey of the present investigation, in the next section, material and method adopted for carrying out research is presented.

\section{Material and Method}

The framework of present investigation is depicted in Figure 1.

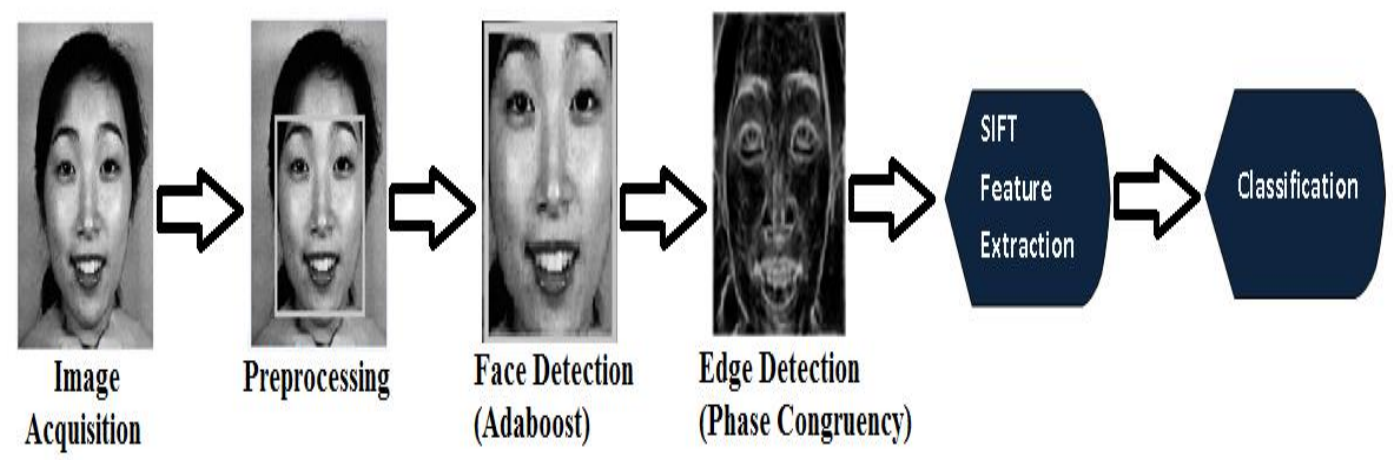

Figure 1. Framework for Present Investigation

\subsection{Image Acquisition}

Firstly, the images are acquired from the database of different person with varying expressions. The performance of the present work is tested on different images of JAFFE and CK database. JAFFE was tested at psychology Department in Kyushu University by Michael Lyons, Miyuki K., and Jori Gyoba. The database contains gray scale images of 10 Japanese female models with a total of 213 images. CK database includes 486 sequences from 97 posers. Each sequence begins with a neutral expression and proceeds to a peak expression. Figure 2 shows the sample images of the JAFFE and CK databases.

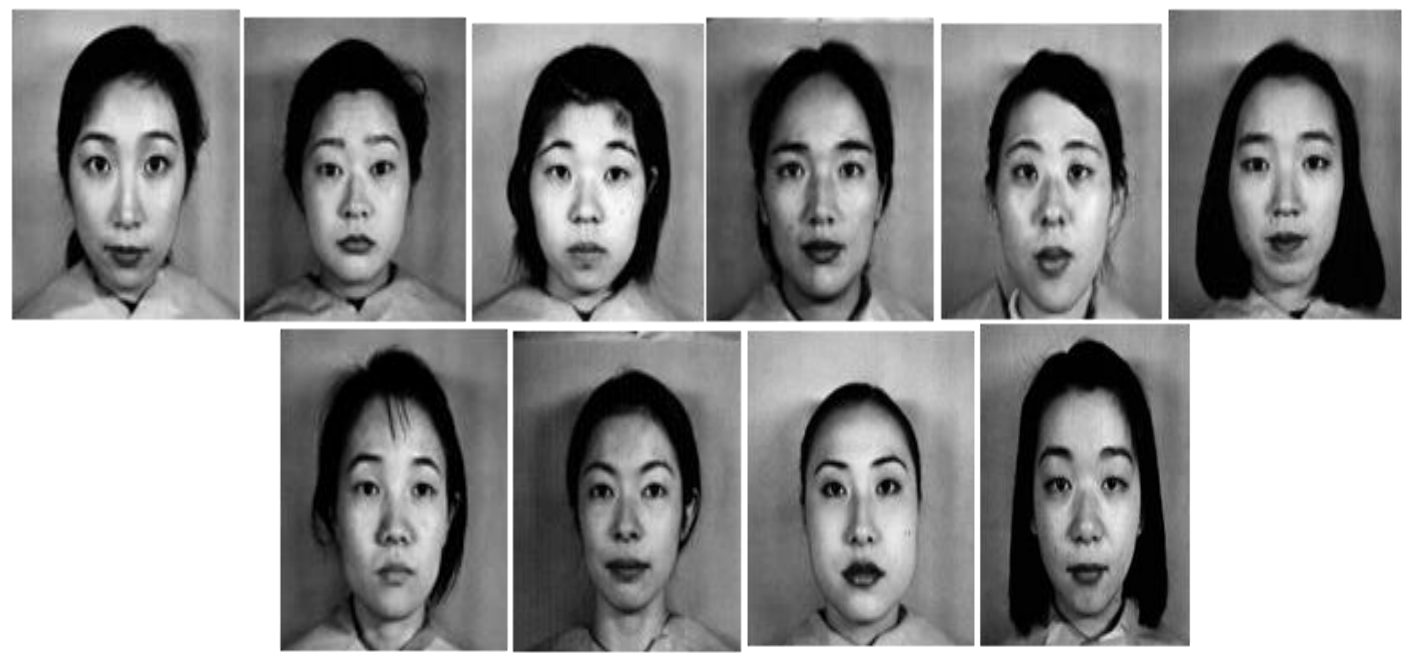

Figure 2. (a) Sample Images of JAFFE Database 


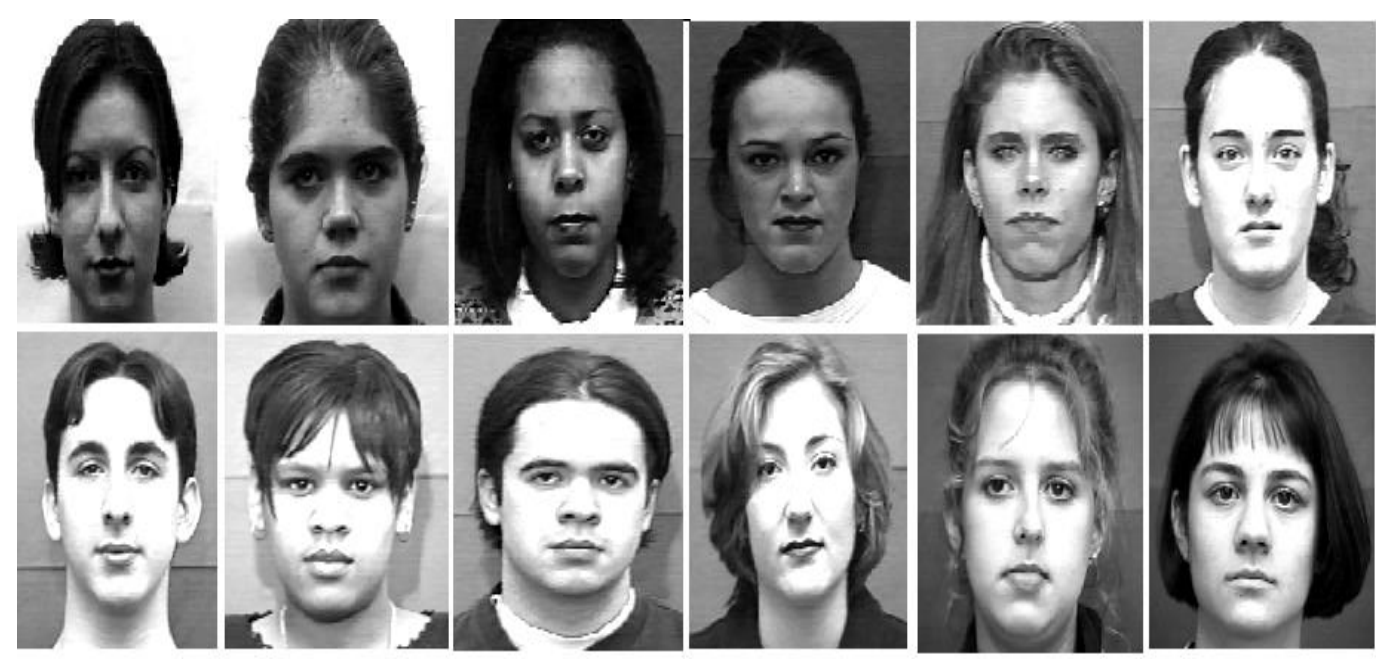

Figure 2. (b) Sample Images of CK Database

\subsection{Preprocessing of Image}

Pre-processing is done to improve the performance of the system. In the present investigation, the test image may have different size from the training set, so each of the input face images are cropped, scaled and normalized to improve the performance of the recognition system. Figure 3 shows the cropped images for different expressions.
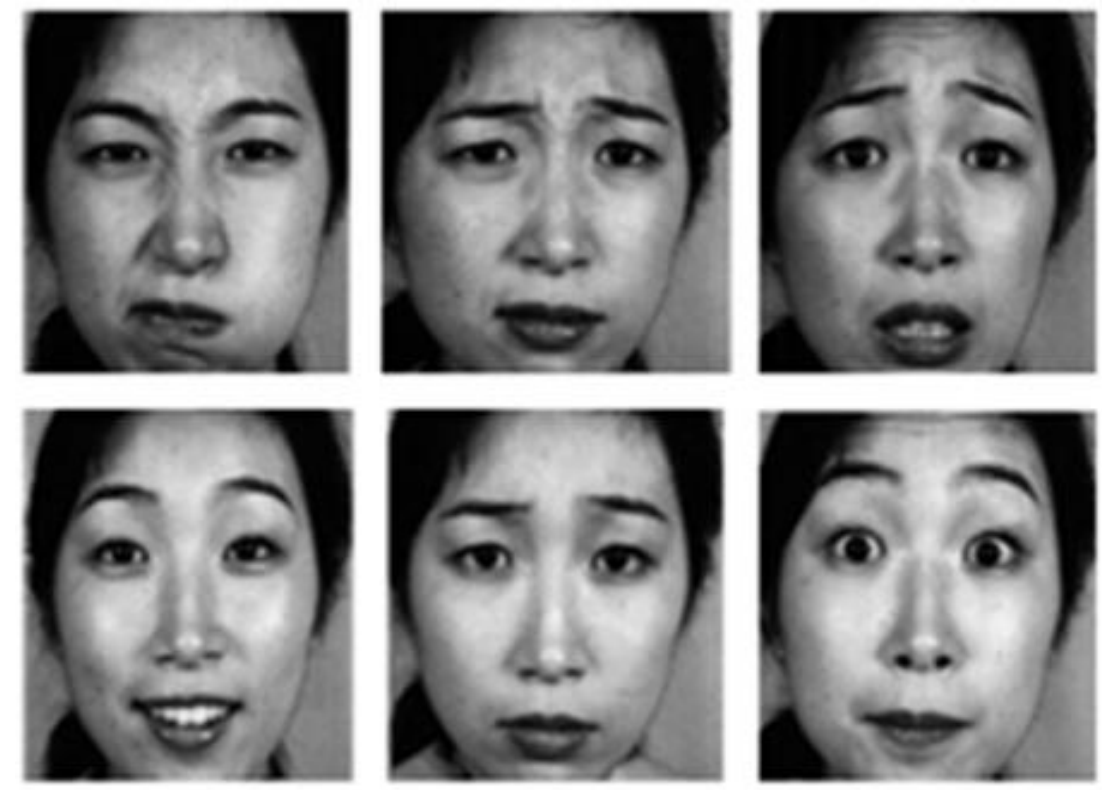

Figure 3. Cropped Faces of Subject 1 of JAFFE Dataset

\subsection{Face Detection}

In this study, Adaboost Viola and Jones algorithm is used for face detection which is most successful technique for real time systems. The three main contributions of VoilaJones face detector are: Haar-like features which represent the image as 'Intergral Image'. The second contribution is AdaBoost learning algorithm which gives a large set of features for fast classification. The third contribution is cascade classifier which enhances weak classifier by combining complex classifier in cascade to enhance the speed of detector. 
In this work Viola Jones approach is used as it detects faces with high speed and also system implementation is easy [18].

\subsection{Edge Detection}

Finally a database matrix is created for a training image and the test image. An edge detection technique is applied on the generated matrix. The main goal of edge detection is to determine and detect sharp discontinuities in an image. Particularly in images, phase gives more information than magnitude, also phase is more immune to noise and distortions, so the use of phase congruency (PC) technique for edge detection is done to improve the efficiency of the system [14] The system implements the work by Kovesi, who further modified the work in two dimensions via 2-D log Gabor with different scales and orientations. In present work, phase congruency model with 2-D log Gabor filter for edge detection is used. According to [20] the phase congruency is given using eq.(1):

$$
P C(x)=\frac{\sum_{\theta} \sum_{s} W_{o}(x)\left|\left[A_{s \theta}(x) \Delta \varphi_{s \theta}(x)-T_{\theta}\right]\right|}{\sum_{\theta} \sum_{s} A_{s \theta}(x)+\varepsilon}
$$

Where $s$ and $\theta$ denotes the index over scale and orientation respectively. $A_{s \theta}(x)$ denotes the amplitude of the filter pair at $x$ and $\Delta \varphi_{s \theta}$ is the phase deviation. $\varepsilon$ denotes the small positive number, used to avoid division with zeros, and its value is approximately 0.001 . Also $\varepsilon$ and $T$ are introduced to reduce noise.

In this investigation, 2D $\log$ Gabor filter is used for feature detection and after that two algorithms for smoothing are used to improve the accuracy of the image [16-17] The LogGabor transfer function is given using eq.(2):

$$
G(w)=\exp \left(\frac{-\left(\log \left(\frac{w}{w_{0}}\right)\right)^{2}}{2\left(\log \left(\frac{k}{w_{0}}\right)\right)^{2}}\right)
$$

Where $w_{0}$ is the filter's centre frequency. The 2D log-Gabor is constructed with the cross-section of the transfer function in the angular direction being a Gaussian function is given using eq.(3):

$$
G(\theta)=\exp \left(\frac{-\left(\theta-\theta_{0}\right)^{2}}{2 \sigma_{\theta}^{2}}\right)
$$

where $\theta_{0}$ represents the orientation of the filter, $\sigma_{\theta}$ is the standard deviation of this Gaussian function. In this study, four scales are denoted by $s$ and six orientations are denoted by $\theta$. Figure 4 shows the output of edge detection on JAFFE database. 

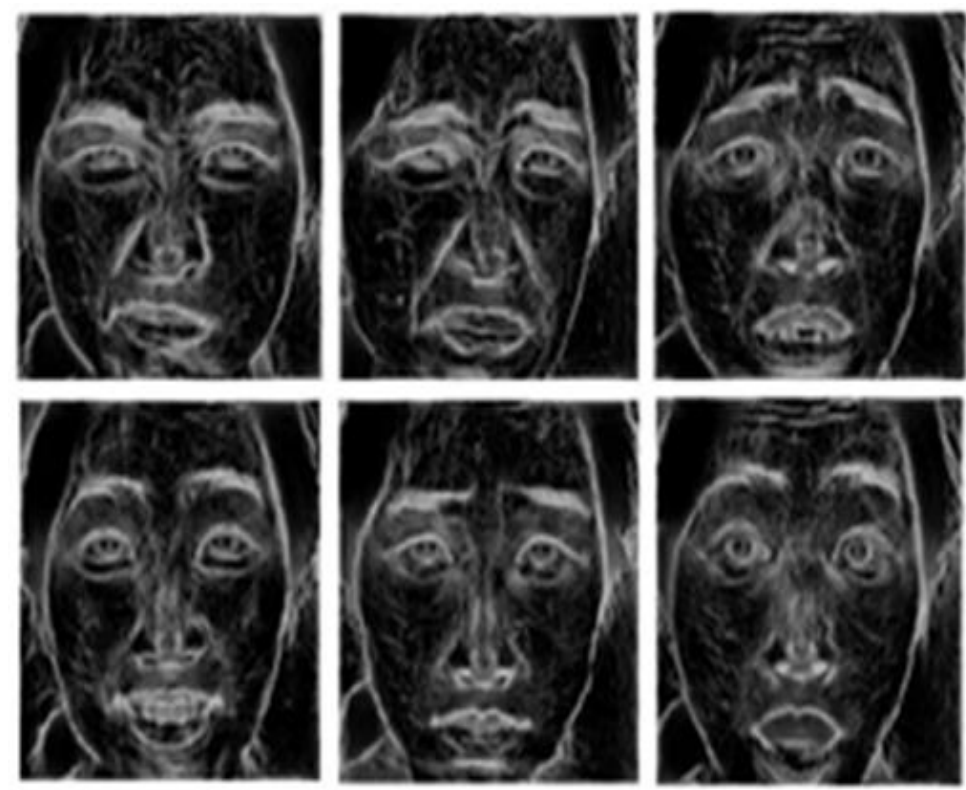

Figure 4. Phase Congruent Features of JAFFE Dataset

\subsection{Feature Extraction}

After the edge detection, facial features are extracted from the face image. Scale Invariant Feature Transform is used as the feature extraction technique, where SIFT features posses a strong robustness to illumination, pose and occlusion variations [18]. Feature extraction involves reduction of amount of resources required to explain a large set of data. Various algorithms have been introduced in literature by many researchers e.g. PCA [10], Independent Analysis component (ICA) [22], Linear Discriminate Analysis (LDA) [23], Gabor transform etc for feature extraction. The author in [11] introduced SIFT for feature extraction which was successfully applied for recognition as it gave better results than PCA, LDA, ICA and other methods discussed in literature. SIFT features are invariant to rotation, scaling, and illumination changes and are robust to noise $[12-13,24]$. The framework for feature extraction using SIFT is discussed as follows:

\section{Step 1: Construction of DOG Scale Space}

The objective is to eliminate unnecessary and wrong details of the image using Gaussian Blurr filter. Gaussian Blurr function is applied to the image with different scales to produce Gaussian images. Then the scale space is constructed by Difference of Gaussian (DOG) images, where DOG is the difference of every two Gaussian images with nearby scale.

The function $L(x, y, \sigma)$ is obtained by convolution of Gaussian function $G(x, y, \sigma)$ and image $I(x, y)$. The function $L(x, y, \sigma)$ is given using eq. (4).

$$
L(x, y, \sigma)=G(x, y, \sigma) * I(x, y)
$$

Where Gaussian function is expressed using eq. (5):

$$
G(x, y, \sigma)=\frac{1}{2 \pi \sigma^{2}} e^{\frac{x^{2}+y^{2}}{2 \sigma^{2}}}
$$

$x$ and $y$ are location coordinates, and $\sigma$ is scale parameter. 
Difference of Gaussian function $D(x, y, \sigma)$ can be given using eq.(6):

$$
D(x, y, \sigma)=(G(x, y, k \sigma)-G(x, y, \sigma)) * I(x, y)=L(x, y, k \sigma)-L(x, y, \sigma)
$$

\section{Step 2: Key-Point localization}

At the position of the point of interest, Taylor series expansion function is used to determine the accurate location and scale of the key-points.

Points of low contrast (sensitive to noise) can be removed by solving magnitude of DOG at each candidate key-point and compare its value with a threshold. If the pixel value of DOG image below threshold, that means low contrast and key-point is discarded.

Secondly to remove edges, discard the flat region and find corners. Two gradients should be calculated which are perpendicular to each other at each key-point. When both gradients are big, point is selected as key-point [12-13].

\section{Step 3: Orientation Assignment to the Key-Points}

To determine the key-point orientation, the gradient magnitude and directions should be calculated around each key-point. Eq. (7) and (8) are used to compute the gradient orientation and magnitude respectively.

$$
\begin{gathered}
\theta(x, y)=\tan ^{-1} \frac{L(x, y+1)-L(x, y-1)}{L(x+1, y)-L(x-1, y)} \\
m(x, y)=\sqrt{(L(x+1, y)-L(x-1, y))^{2}+(L(x, y+1)-L(x, y-1))^{2}}
\end{gathered}
$$

where $L$ is a Gaussian smoothed image with closest scale to that of a key-point.

\section{Step 4: SIFT Feature Generation}

To eliminate orientation and illumination problems, each key-point is assigned with the 128 elements. Firstly, a $16^{*} 16$ window is selected around the key-point where sixteen $4^{*}$ 4 window are generated from the above window. Magnitude and orientation are computed for each $4 * 4$ window and draw the histogram. Finally, histogram is further divided into 8 bins and finally each key-point is represented by 128 elements [12-13, 18].

\subsection{Classification}

The last step is the classification of the extracted features, features from test images and testing images are compared and recognition is achieved. In last two decade researchers have given a number of classifiers for analysis of the work [25-26] such as PCA, fisher linear classifier, K-NN, K-PCA, SVM and classifiers based on statistical learning etc.

\section{Support Vector Machine (SVM)}

SVM is a supervised learning method [26], and is from a family of generalized linear classifier. SVM is the strongest of all the classifiers used in literature specially for face recognition. It has a special property that, it simultaneously maximizes the geometric margin and minimizes the empirical classification error. In SVM algorithm, a hyperplane is used to separate the different classes. The aim of SVM is to find the optimal hyperplane for classification purpose [25-27]. 


\section{Experimental Results}

The performance of the present work is evaluated on the JAFFE and CK databases. Feature extraction is done by a hybrid technique: Phase Congruency and Shift Invariant Feature Transform (SIFT). For classification linear classifier SVM and K-Means are used. To evaluate the performance of the work, comparison is done by applying phase congruency- shift invariant feature transform technique and applying only shift invariant feature transform with both the classifiers.

Comparisons has being shown in Table 2 where the output of SVM and K-Means classifiers are shown by applying PC-SIFT and SIFT technique on a number of subjects for different expressions. After the analysis it is observed that the recognition rate is maximum with SVM classifier applied on different expression images. When features are extracted using PC-SIFT approach along with SVM classifier gives better recognition accuracy for both the datasets then SIFT technique in all the cases.

Table 2. Comparison of Recognition Rate on JAFFE and CK Dataset

\begin{tabular}{|c|c|c|c|c|c|c|c|c|}
\hline \multirow{2}{*}{\begin{tabular}{c} 
Database \\
\multirow{2}{*}{$\begin{array}{c}\text { Expressio } \\
\mathrm{n}\end{array}$}
\end{tabular}} & \multicolumn{3}{|c|}{$\begin{array}{c}\text { SVM } \\
\text { (\%Accuracy })\end{array}$} & \multicolumn{2}{c|}{$\begin{array}{c}\text { k-Means } \\
\text { (\%Accuracy }\end{array}$} & \multicolumn{2}{c|}{$\begin{array}{c}\text { SVM } \\
\text { (\%Accuracy) }\end{array}$} & \multicolumn{2}{c|}{$\begin{array}{c}\text { k-Means } \\
\text { (\%Accuracy) }\end{array}$} \\
\cline { 2 - 10 } & PC-SIFT & SIFT & $\begin{array}{c}\text { PC- } \\
\text { SIFT }\end{array}$ & SIFT & $\begin{array}{c}\text { PC- } \\
\text { SIFT }\end{array}$ & SIFT & PC-SIFT & SIFT \\
\hline Anger & 94 & 85 & 64.8 & 60.2 & 92.5 & 82.2 & 61.4 & 60.1 \\
\hline Disgust & 92.1 & 86.5 & 65.81 & 53.89 & 90.2 & 84.3 & 63.48 & 59.3 \\
\hline Fear & 93.9 & 84.5 & 64.05 & 57.36 & 95.6 & 85.5 & 65.6 & 58.1 \\
\hline Happy & 90.5 & 79 & 62.9 & 56.2 & 91 & 82.1 & 60.1 & 57 \\
\hline Sad & 92.8 & 83.8 & 63.9 & 55.9 & 89.7 & 79.2 & 64.3 & 58.7 \\
\hline Surprise & 91.3 & 84.6 & 70.6 & 61.3 & 90.1 & 84.1 & 68.5 & 62 \\
\hline
\end{tabular}

It is obvious from Table 2 for JAFFE database PC-SIFT gives highest $92.4 \%$ recognition accuracy with SVM classifier while SIFT gives $83.9 \%$ recognition accuracy and K-Means classifier gives a recognition rate of $65.3 \%$ whereas with SIFT the recognition rate is $57.4 \%$. For CK database, proposed approach gives the accuracy of 91.5\% for PC-SIFT and $82.9 \%$ for SIFT using SVM and $63.8 \%$ and $59.2 \%$ for PC-SIFT and SIFT respectively for K-Means classifier. Hence the proposed technique PC-SIFT outperform SIFT features for different expressions.

\section{Comparison with State-of-art Algorithms}

From the JAFFE dataset, neutral image is chosen as the reference image per person and rest of the images as test images. In Figure 5, the result obtained from the proposed work is compared with other techniques. The recognition rate of state-of-art algorithms when compared with proposed technique PC-SIFT is found to be better in comparison with the remaining techniques on JAFFE dataset. 


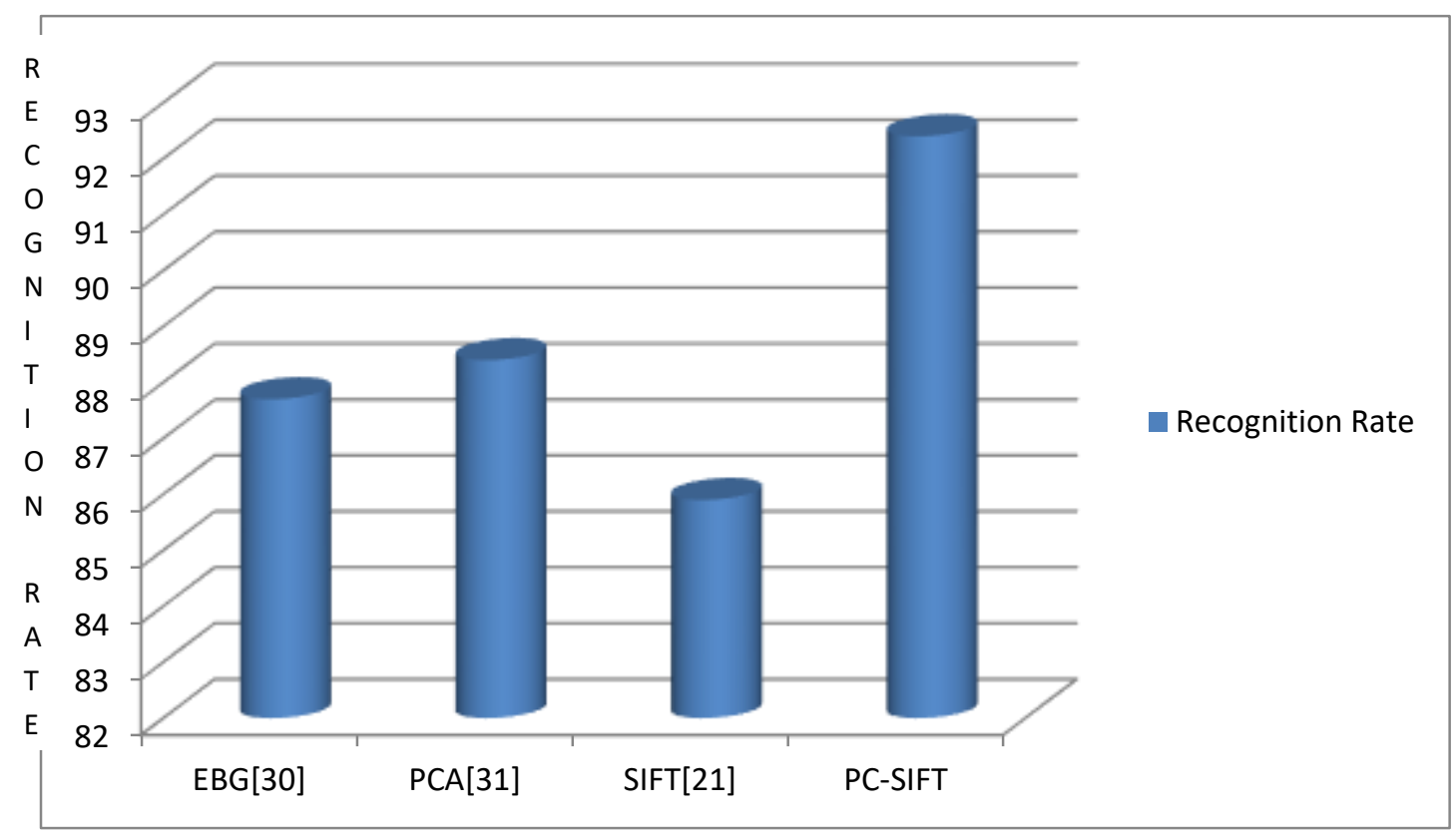

Figure 5. Performance Comparison on JAFFE Dataset

Figure 5 present the performance of the listed methods in form of recognition rates for changeable expressions. The recognition rates of EBG and PCA are $87.8 \%$ and $88.4 \%$ respectively. The original SIFT has an accuracy of $85.9 \%$ which is lowest of all state-ofart techniques. The proposed PC-SIFT have the recognition rate of $92.4 \%$. It is evident from Figure 4 that the result obtained from proposed technique PC-SIFT is better in comparison with the recognition performance of remaining techniques.

\section{Conclusion}

In the present investigation, PC-SIFT is used as a hybrid technique which exploits the advantages of both PC and SIFT techniques. PC is used to enhance the edges of the image and SIFT features posses a strong robustness to illumination, pose and occlusion variations, finally performance of the technique is evaluated by SVM classifier. It is observed that PC-SIFT outperforms SIFT technique and gives an average recognition accuracy of $92.4 \%$ and $91.5 \%$ respectively for different expression using SVM classifier for JAFFE and CK dataset. A number of experiments conducted in the work shows that the proposed method works well on several images of JAFFE and CK database. The work presented here has a significant contribution to computer vision, public security and various other fields.

\section{References}

[1] Phillips P. J., Moon H., Rauss P. J., and Rizvi S. A., "The FERET Evaluation Methodology for Face Recognition Algorithms," IEEE Transactions on Pattern Analysis and Machine Intelligence, vol. 22, no. 10, (1995), pp. 1090-1104.

[2] Zhao W., Chellappa R., Rosenfeld A., Phillips P.J.,"Face Recognition: A Literature Survey”, ACM Computing Surveys, vol. 35, no. 5, (2003), pp. 399-458.

[3] Moon H. "Biometrics Person Authentication Using Projection-Based Face Recognition System in Verification Scenario," International Conference on Bioinformatics and its Applications, Hong Kong, China, (2006), pp.207-213.

[4] Jafri R., Arabnia H. R., "A Survey of Face Recognition Techniques", Journal of Information Processing Systems, vol.5, no. 2, (2009), pp. 41-68.

[5] Turk M. and Pentland A.,"Eigen faces for recognition”, Journal of Cognitive Neuroscience, vol.3, no.1, (1991), pp.71-86. 
[6] Lee Yongjin, Lee Kyunghee, and Pan Sungbum, "Local and global feature extraction for face recognition", In Audio-and Video-Based Biometric Person Authentication, Springer Berlin Heidelberg, (2005), pp. 219-228.

[7] Belhumeur P. N., Hespanha J. P. and Kriegman D. J., "Eigenfaces vs Fisherfaces: Recognition using class specific linear projection”, IEEE Trans. Pattern Analysis and Machine Intelligence, vol.19, no.7, (1997), pp.711-720.

[8] Zhou C., Wei X. and Zhang Q. "Face recognition based on HMM-SVM", ICIC Express Letters, vol.1, no.2, (2007), pp.137-143.

[9] Ojala T., Pietikainen M. and Maenpaa T., "Multi-resolution gray-scale and rotation invariant texture classification with local binary patterns", IEEE Trans. Pattern Analysis and Machine Intelligence, vol.24, no.7, (2002), pp. 971-987.

[10] Sakthivel S., Lakshmipathi R.,'Enhancing Face Recognition using Improved Dimensionality Reduction and feature extraction Algorithms-An Evaluation with ORL database", International Journal of Engineering Science and Technology, vol. 2, no. 6, (2010), pp. 2288-2295.

[11] Lowe D., "Distinctive image features from scale-invariant key-points", Int. Journal of Computer Vision, vol.60, no.2, (2004), pp. 91-110.

[12] Ke Y. and Sukthankar R., "PCA-SIFT: A more distinctive representation for local image descriptors", Proc. of IEEE Conf. Computer Vision and Pattern Recognition, vol.2, (2004), pp. 506-513.

[13] Lin S. D. , Lin J. , and Chiang C., "Using gradient features from scale-invariant key-points on face recognition. " International Journal of Innovative Computing, Information and Control, vol. 7, no. 4, (2011), pp. 1639-1649.

[14] Skarbnik N., Zeevi Yehoshua Y. and Sagiv Chen "The Importance of phase in image processing" PhD diss., Technion-Israel Institute of Technology, Faculty of Electrical Engineering, (2009).

[15] Morrone M. C. and Burr D. C., "Feature detection in human vision: A phase-dependent energy model". Proc. R. Soc. London. B, vol. 235, no. 1280, (1988), pp. 221-245.

[16] Venkatesh S. and Owens R. A., "An energy feature detection scheme", International Conference on Image Processing, (1998), pp. 553-557.

[17] Kovesi P. D., "Image features from phase congruency". Videre: Journal of Computer Vision Research, vol. 1, no. 3, (1999), pp. 1-26.

[18] Viola P., and Jones Michael J., "Robust real-time face detection.", International Journal of Computer Vision, vol. 57, no.2, (2004), pp. 137-154.

[19] Yuan Xiaoyan, and Pengfei Shi., "Iris feature extraction using 2D phase congruency." In Information Technology and Applications, ICITA 2005, International Conference, vol. 2, (2005), pp. 437-441.

[20] Struc V., Pavesic N., "Phase congruency features for palm-print verification", IET Signal Processing, vol. 3, no. 4, (2009), pp. 258-268.

[21] Wang Yu-Yao, Li Zheng-Ming, Wang Long, and Wang Min,"A scale invariant feature transforms based method." Journal of Information Hiding and Multimedia Signal Processing, vol 4, no 2, (2013), pp.7389.

[22] Hyvärinen Aapo and Oja Erkki, "Independent Component Analysis: Algorithms and Applications", Neural Networks Research Centre Helsinki University of Technology P.O. Box 5400, Finland, Neural Networks, 13(4-5), (2000), pp. 411-430.

[23] Lu J., Plataniotis K., and Venetsanopoulos A., "Face recognition using LDA based algorithms", IEEE Transactions on Neural Networks, vol. 14, no. 1, (2003), pp.195-200.

[24] Dagher I., Sallak Nour El, and Hazim Hani, "Face Recognition using the most Representative Sift Images", International Journal of Signal Processing, Image Processing and Pattern Recognition, vol. 7, no. 1, (2014), pp. 225-236.

[25] Burges, Christopher J.C., "A tutorial on support vector machines for pattern recognition", Data mining and knowledge discovery, vol. 2, no. 2, (1998), pp. 121-167.

[26] Vapnik V., "Statistical learning theory", John Wiley and Sons, New York, (1998)

[27] Phillips, Jonathon P., "Support vector machines applied to face recognition", US Department of Commerce, Technology Administration, National Institute of Standards and Technology, vol. 285, (1998).

[28] Križaj J., Štruc V., and Pavešić N., "Adaptation of SIFT features for robust face recognition." Image Analysis and Recognition. Springer Berlin Heidelberg, (2014), pp. 394-404.

[29] Pun Chi-Man , Lee Moon-Chuen, "Extraction of shift invariant wavelet features for classification of images with different sizes", IEEE Transactions on Pattern Analysis and Machine Intelligence, vol. 26, no. 9, (2004), pp. 1228-1233.

[30] X.Xie, K. Lam, "Face recognition using elastic local reconstruction based on a single face image", Pattern Recognition, (2008), 41 pp. 406 - 417.

[31] Luo Juan, and Oubong Gwun, "A Comparison of SIFT, PCA-SIFT and SURF”, International Journal of Image Processing, vol. 3, no. 4, (2015), pp. 143-152. 


\section{Authors}

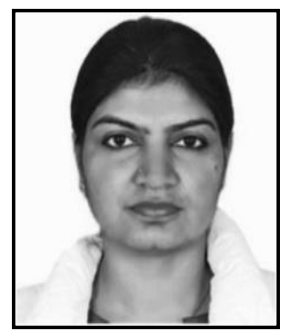

Deepti Ahlawat, Received her M.Tech degree in 2008 from N.C. College of Engg., Israna, Panipat under Kurukshetra University, Kurukshetra, India. She is now a Ph.D. scholar at BPSMV,Khanpur Kalan, Sonipat, India. Her research interest includes Pattern recognition and Image Processing.

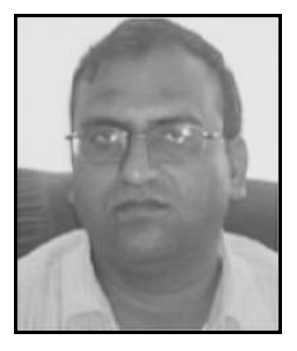

Vijay Nehra, earned Ph.D degree in Electronics and Communication Engineering in 2009 from Maharshi Dayanand University, Rohtak. He is currently working as professor in the Electronics and Communication Engineering Department at the Faculty of Engineering and Technology, Bhagat Phool Singh Mahila Vishwavidayala, Khanpur Kalan, Sonipat, Haryana, India. Dr. Nehra has a professional experience of 14 years in teaching, research, curriculum planning, laboratory development, educational administration, planning, management and execution. He has over 45 publications in refereed journals and proceedings along with many articles in the field of engineering and engineering education. $\mathrm{He}$ is a life member of various professional societies such as ISTE, CSI, IETE, Institution of Engineers, Plasma Science Society of India. 
International Journal of Signal Processing, Image Processing and Pattern Recognition Vol. 10, No. 9 (2017) 